Revue de l'association française de recherche sur

I'histoire du cinéma

$61 \mid 2010$

Aux sources du burlesque cinématographique : les comiques français des premiers temps

\title{
Drôle de boxe. Stratégies du rire dans le film comique français au début des années 1910
}

\section{Frank Kessler}

\section{(2) OpenEdition}

1 Journals

Édition électronique

URL : https://journals.openedition.org/1895/3840

DOI : 10.4000/1895.3840

ISBN : 978-2-8218-0980-2

ISSN : $1960-6176$

Éditeur

Association française de recherche sur l'histoire du cinéma (AFRHC)

Édition imprimée

Date de publication : 1 septembre 2010

Pagination : 191-204

ISBN : 978-2-913758-62-9

ISSN : 0769-0959

\section{Référence électronique}

Frank Kessler, « Drôle de boxe. Stratégies du rire dans le film comique français au début des années 1910 », 1895. Mille huit cent quatre-vingt-quinze [En ligne], 61 | 2010, mis en ligne le 01 septembre 2013, consulté le 16 avril 2022. URL : http://journals.openedition.org/1895/3840 ; DOI : https://doi.org/ $10.4000 / 1895.3840$ 


\section{Drôle de boxe. Stratégies du rire dans le film comique français au début des années $1910^{1}$}

par Frank Kessler

Dans la série d'articles confiés par Victorin Jasset en novembre 1911 à la revue Ciné-Journal pour proposer une "Étude sur la mise en scène », on peut lire cette remarque quelque peu étonnante et qui fait référence à la situation du cinéma français vers la fin de 1909 : "André Deed avait fait école, presque en même temps que Max Linder, son précurseur. " ${ }^{2}$ Cette affirmation est en effet tout à fait surprenante, ou du moins elle va à l'encontre de ce que l'on dirait intuitivement : on s'attendrait bien sûr à lire exactement l'inverse. Et en plus, s'il y a un grand comique dont Linder, aujourd'hui, est considéré être le précurseur, c'est bien Charlot, mais très certainement pas André Deed. Par ailleurs, d'un point de vue en quelque sorte normatif, on peut se demander comment Jasset, en 1911, avait pu se méprendre à ce point sur les qualités respectives des deux comiques, étant donnée la perspective téléologique qui est la sienne dans ces pages, prônant avant tout l'évolution de l'art cinématographique vers le réalisme ${ }^{3}$. À cela s'ajoute le fait non seulement que la carrière cinématographique de Deed commence bien avant celle de Linder, mais que le personnage de Boireau est créé un peu avant celui de Max ${ }^{4}$. Cette observation, qui apparaît si contre-intuitive aujourd'hui, nous

1 L'auteur tient à remercier Sabine Lenk pour sa lecture critique de ce texte et pour ses précieux commentaires et suggestions.

2 Victorin Jasset, " Études sur la mise en scène » dans Marcel Lapierre, Anthologie du cinéma, Paris, La Nouvelle Édition, 1946, p. 95. Toutefois, étant donné le caractère largement stratégique de cet série d'articles, il faudrait peut-être aussi reconstruire la visée dans laquelle Jasset prononce cette observation. 3 La dernière phrase de cette série d'articles le dit en toutes lettres à propos des comédies de la Vitagraph : «Cependant, tout cela était nécessaire pour pouvoir donner au public la complète illusion du réel." "

4 Pour les deux comiques, voir Jean A. Gili, André Deed, Genoa Bologna, Edizioni la Mani/Cineteca di Bologna, 2005 ; Maud Linder, Les Dieux du cinéma muet. Max Linder, Bruxelles/Paris, Éditions Atlas, 1992: Maud Linder, Max Linder était mon père, Paris, Flammarion, 1992; Max Linder. Ein früher Star (sous la direction de Thomas Kuchenbuch), Vienne, Cologne, Weimar, Böhlau Verlag, 2008 ( = Maske und Kothurn 54, 1-2). 


\section{BOIREAU ROI DE LA BOXE}

$1895 /$

$n^{\circ} 61$

septembre

2010

192

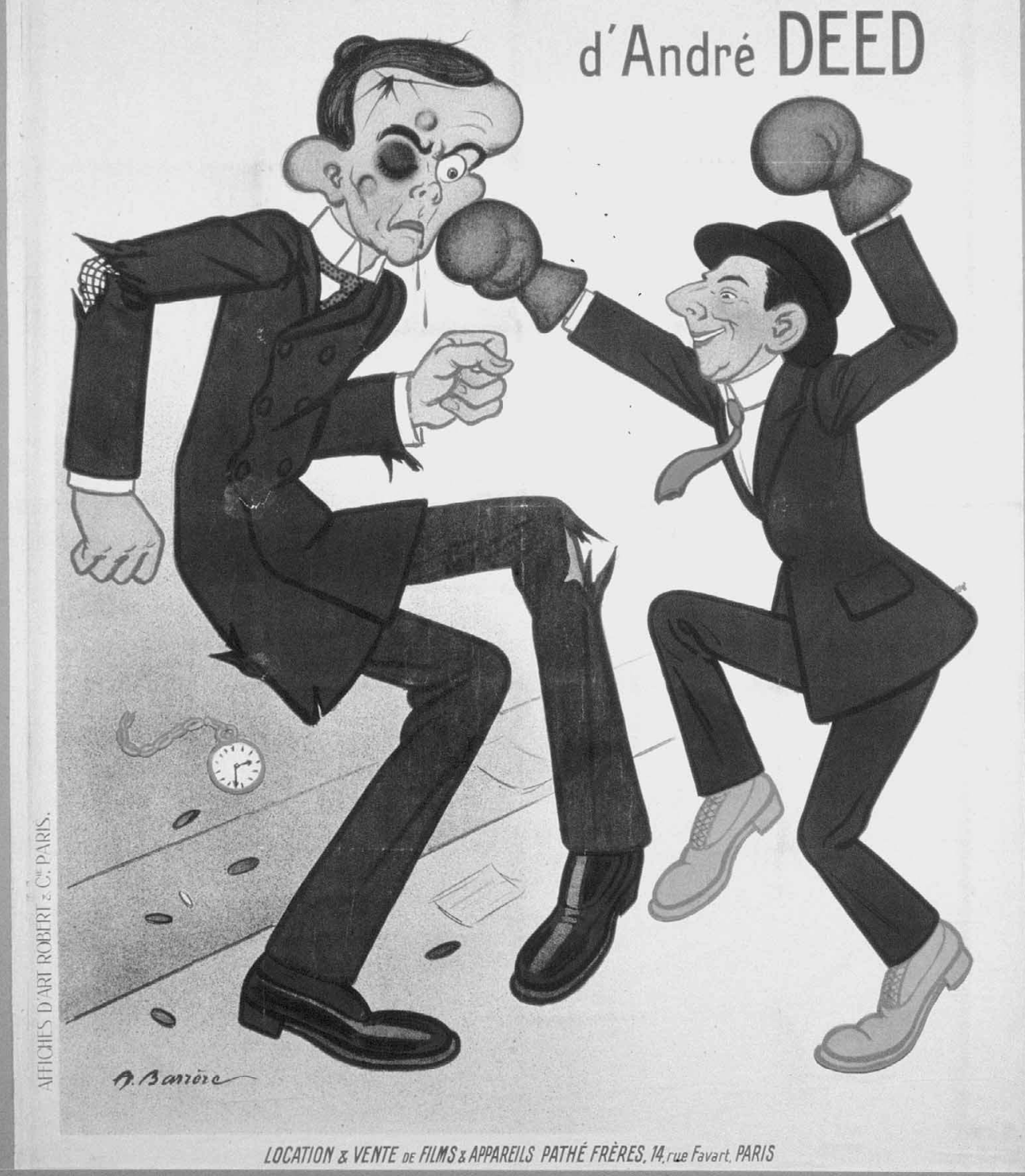

Boireau, roi de la boxe, Pathé, 1912. 
invite alors à interroger de manière plus fondamentale la conception « évolutionniste » que l'on rencontre si souvent dans les études sur les comiques des premiers temps.

II y a, bien évidemment, des différences qui, presque littéralement, sautent aux yeux. Jean Mitry, dans son grand ouvrage sur I'histoire du cinéma, en rend compte en proposant une distinction entre un " comique des faits » et un " comique des situations » - le passage de l'un à l'autre étant le mérite, selon Mitry, de Max Linder notamment - ces deux formes ayant été précédées par un comique fondé sur des « culbutes grotesques »5. Francis Lacassin, de son côté, propose une véritable périodisation, allant d'une " période préhistorique (1896-1905) " à une phase "formative (1906-1911) », suivie par une période « classique (1912-1914) », ainsi que deux formes majeures, un " comique d'observation " pratiqué notamment par Louis Feuillade et Max Linder, ainsi qu'un « comique de mouvement » dont Roméo Bosetti et Jean Durand sont les représentants ${ }^{6}$. Or, ce qui frappe quand on regarde la production du début des années 1910, par exemple, c'est le fait que différents types de films comiques semblent plutôt co-exister. Autrement dit, de telles périodisations reflètent moins un état de fait qu'une perspective permettant alors de qualifier, pour une période donnée, certains comiques comme étant soit « en avance », soit « en retard " par rapport au style dominant".

Or, dans la production Pathé vers 1912-1913, pour rester avec Deed et Linder, les personnages de Boireau et de Max, tout différents qu'ils sont l'un de l'autre, font tous les deux partie de la gamme de films comiques proposés par cette firme au public. En effet, loin de considérer Deed comme un acteur démodé, la maison du coq vient de le réintégrer dans les rangs de ses vedettes, annonçant et célébrant son retour d'Italie par la bande Gribouille redevient Boireau (1912). En accord avec sa stratégie commerciale jusque-là fort profitable, Pathé continue apparemment à miser sur la diversification et la variation de l'offre dans ses catalogues, permettant ainsi aux exploitants de composer des programmes en fonction des préférences de leur clientèle. Même s'il y a pu avoir, à ce moment-là, des différences par rapport à la popularité des diverses vedettes travaillant pour Pathé, la firme a dû voir un potentiel commercial pour le type d'acteur burlesque qu'est André Deed, car autrement on n'aurait guère investi dans la relance du comique sur le marché français.

5 Voir Jean Mitry, Histoire du cinéma - Art et industrie II. 1915-1925, Paris, Éditions Universitaires, 1969, p. 42 (pour les « culbutes grotesques ») et surtout p. 48. Mitry ne construit cependant pas ces catégories de manière théorique.

6 Francis Lacassin, Pour une contre-histoire du cinéma, Paris, UGE, coll. 10/18, 1972, p. 76. Cette périodisation est reprise par Éric Le Roy, " Max Linder, der Mann mit dem seidenen Hut », dans Max Linder. Ein früher Star, op. cit., pp. 52-59.

7 Dans un travail antérieur avec Sabine Lenk, nous avons proposé à un niveau plus général, pour le cinéma des premiers temps, une distinction entre films purement attractionnels (comme par exemple les 
$1895 /$

\section{Coups de poings burlesques}

Depuis les débuts du spectacle des vues animées, la boxe a été l'un des sujets les plus populaires, aux États-Unis comme en Europe, où ce sport comme sa représentation cinématographique ont rapidement suscité l'intérêt du public ${ }^{8}$. Entre 1911 et 1913, au moins quatre films comiques produits par Pathé intègrent le pugilat dans leur trame narrative.

Voici la description donnée dans le Bulletin Pathé pour Little Moritz demande Rosalie en mariage :

Little Moritz s'éprend de Rosalie et la demande en mariage. Mais, paralysé par une invincible timidité, il n'a pas l'heur de plaire au père de sa bien-aimée qui l'expulse, en déclarant qu'il ne donnera sa fille qu'à un homme énergique. Little Moritz ne se tient pas pour battu et, soulevé par un courage de désespéré, il apprend l'art de devenir énergique. Grâce à la méthode

numéros d'acrobatie, de prestidigitation etc. reproduits devant la caméra), des films mettant l'accent sur le mouvement (films de course-poursuite etc.) et des films mettant l'accent sur l'action dramatique pour désigner trois régimes de jeu corporel. Nous avons, cependant, évité de combiner cette tripartition avec une périodisation, car dans la pratique ces différents types de jeu co-existent peu ou prou pendant la plus grande partie de cette période. Voir Frank Kessler, Sabine Lenk, "Cinéma d'attractions et gestualité ", in les Vingt premières années du cinéma français (sous la direction de Jean A. Gili, Michèle Lagny, Michel Marie et Vincent Pinel), Paris, Presses de la Sorbonne Nouvelle, 1995, pp. 195-202.

8 Voir Dan Streible, Fight Pictures : A History of Boxing and Early Cinema, Berkeley, University of California Press, 2008. Par ailleurs, les combats de boxe figurent parmi les premières formes longues du spectacle cinématographique. En plus, la boxe continue bien évidemment d'être un sujet dans des films comiques. Que I'on pense, pour citer un exemple bien plus tardif, à City Lights de Charles Chaplin (1931). 


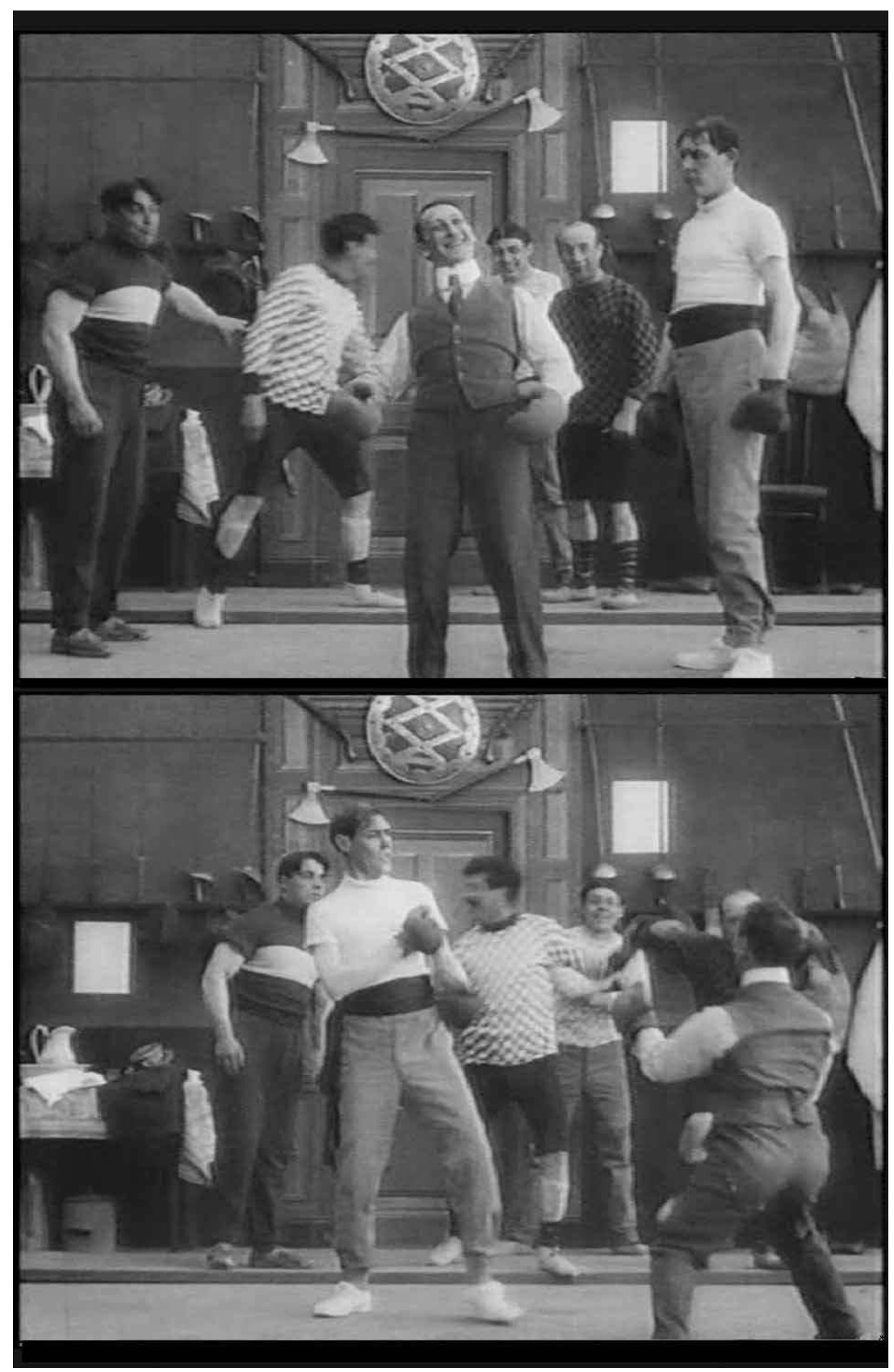

Fig. 1. Little Moritz demande Rosalie en mariage, Comica, 1911. 
du professeur Obujousky, le prétendant évincé, maintenant fort comme un lion, terrorise par son audace et sa force son futur beau-père qui lui accorde, non sans trembler, la main de sa fille. ${ }^{9}$

La boxe, ici, sert alors en premier lieu à déchaîner l'énergie agressive du héros et n'est curieusement pas mentionnée dans cette description du film. L'obscure " méthode du professeur Obujousky " se révèle pourtant être tout simplement un entraînement à ce sport (fig. 1). Après avoir reçu trois fois des coups qui le mettent par terre, Little Moritz se relève pour passer lui-même à l'attaque. En sautant, il réussit à toucher son adversaire, bien que celui-ci soit beaucoup plus grand que lui, et le fait tomber à son tour. Little Moritz continue à le frapper, et quand les autres essaient de le retenir, il se tourne contre eux aussi. Enlevant ses gants, il s'en va, non sans donner un coup violent au pauvre homme qui, à l'entrée du bâtiment, fait de la publicité pour l'établissement pugilistique. Quand il quitte la salle de sport, l'instructeur assis au sol croise ses bras dans un geste voulant dire quelque chose comme "ça alors !", comme s'il n'arrivait pas à croire ce qui vient de se passer. Une fois arrivé chez Rosalie, Little Moritz frappe le père de celle-ci et se met à casser tout dans l'appartement, jusqu'à ce qu'il obtienne la main de sa bien-aimée.

La boxe même, autrement dit, n'est pratiquée que brièvement dans ce film et sert avant tout à motiver le changement de caractère du héros. Cette scène - ce tableau, pour être plus précis - produit des effets comiques de plusieurs manières. D'une part, les boxeurs eux-mêmes apparaissent plutôt comme des caricatures d'athlètes, avec des mouvements un peu maladroits et un physique qui ne semble pas tout à fait adéquat pour l'exercice sportif. Les échanges de coups apparaissent assez désordonnés et ne suggèrent aucunement la maîtrise du pugilat. D'autre part, il y a un fort contraste entre Little Moritz, son caractère, son costume ainsi que son comportement, et le milieu sportif dans lequel il entre. Le combat d'entraînement est tout à fait clownesque et doit sans doute beaucoup au monde du cirque dans sa mise en scène. Le comique repose alors avant tout sur la tension entre l'univers du sport, à l'arrière-fond, et la manière avec laquelle il est pratiqué par ces personnages. Ce comique est basé sur des mouvements qui apparaissent ridicules, parce que visiblement inadéquats, maladroits et exagérés. Le déchaînement destructeur du personnage par lequel le film se termine n'a plus rien à voir avec la boxe.

Dans Boireau, roi de la boxe (fig. 2), on trouve une trame narrative similaire dans la mesure où c'est également une expérience de rejet qui amène le héros à apprendre la boxe pour

9 Voir Henri Bousquet, Catalogue Pathé des années 1896 à 1914. 1910-1911, Éditions Henri Bousquet, 1994, p. 448. Le film sort en octobre 1911. 


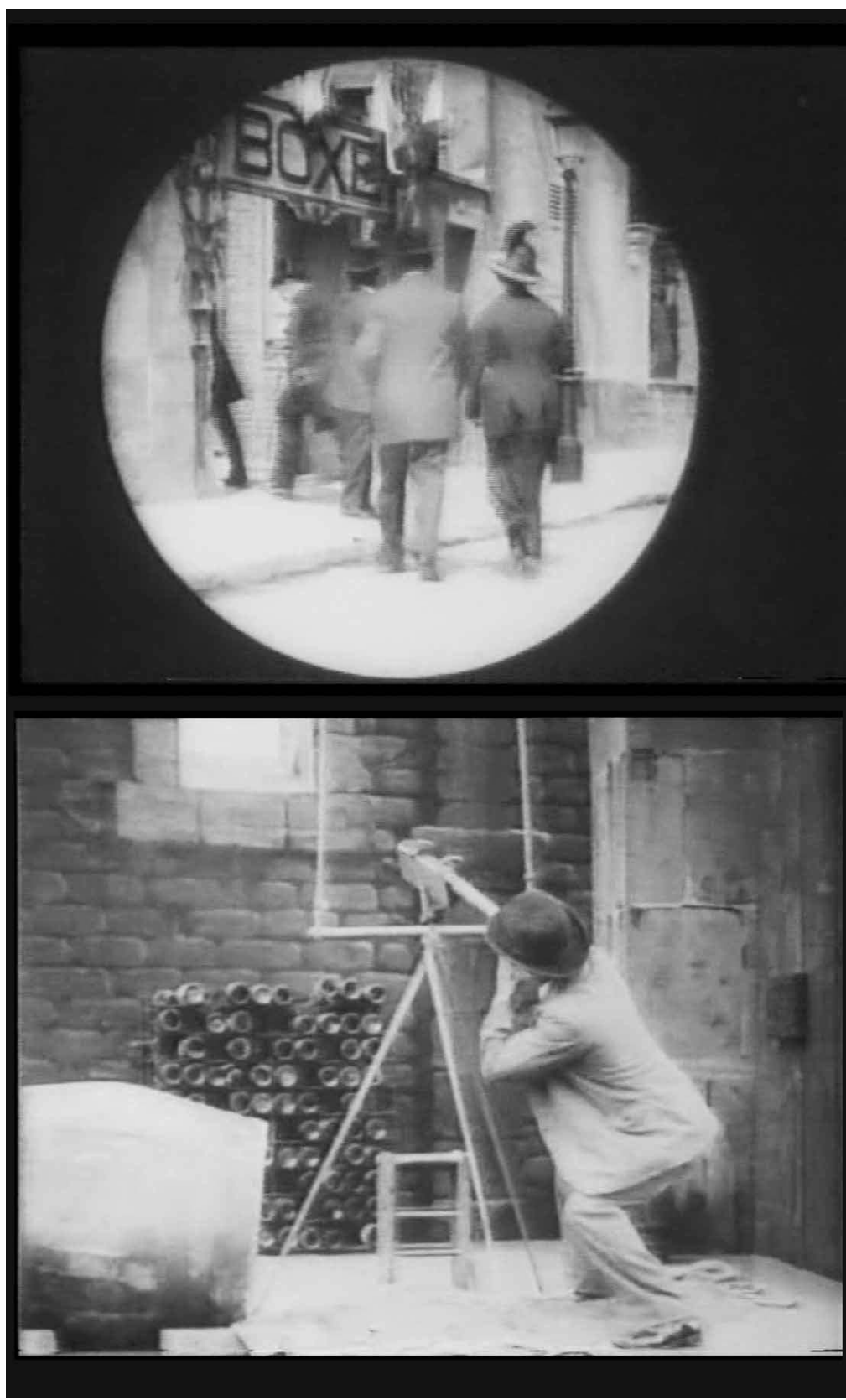

Fig. 2. Boireau, roi de la boxe, Pathé, 1912.

1895

$n^{\circ} 61$

septembre

2010

197

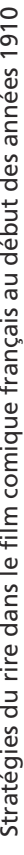

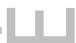


ensuite triompher de ses adversaires, mais sans pratiquer ce sport selon les règles. En voici la description (assez approximative, il faut le dire) dans le Bulletin Pathé :

Boireau emballé par les exploits des Carpentier et des Willie Lewis, veut à son tour devenir champion du monde. Après qu'on lui eut refusé l'entrée dans une salle, puis bafoué et houspillé, notre ami va prendre des leçons. Devenu boxeur émérite il prend une revanche éclatante sur ses adversaires, faisant une " grosse tête » à l'un et un nez " gros comme ça » à un autre. ${ }^{10}$

Dans ce film, le thème de la boxe intervient alors à deux niveaux. Premièrement, Boireau veut aller voir un match et se fait mettre à la porte à cause de son comportement impropre par deux autres spectateurs. Comme Little Moritz dans l'exemple précédent, il va alors prendre une leçon de pugilat dans un établissement d'entraînement. Cette leçon est encore plus courte que celle de Little Moritz. L'entraîneur montre vaguement à Boireau quelques mouvements de coups et attend que celui-ci les imite. Boireau, par contre, le frappe directement et le professeur de boxe se retrouve par terre. Boireau s'en va, les poings toujours gantés, et I'instructeur exécute le même geste que son homologue dans le film avec Little Moritz. Ce film établit donc un contraste avec la pratique "sérieuse " du sport lors du match de boxe dans la première partie, dont on voit quelques images, tandis que Boireau se sert de son entraînement pour frapper d'abord un facteur, qui a le malheur de le heurter dans la rue, et à qui il fait " un nez gros comme ça ». Puis il effraie le gardien devant le gymnase en cassant une lanterne d'un seul coup de poing (fig. 3). Finalement, il prend sa vengeance sur les deux messieurs. L'un se trouve transformé, par la force des coups, en un tas de vêtements et le deuxième finit par avoir une " grosse tête ". Tous ces effets sont réalisés à l'aide de trucages et correspondent à l'univers « incohérent » (pour utiliser un terme de l'époque qui se trouve également dans le titre $d^{\prime}$ un autre film de la série ${ }^{11}$ ) au sein duquel se meut si souvent le personnage de Boireau. Ainsi, au début du film, il prend le journal pour le transformer en gant de toilette, enfile son pantalon à l'aide d'un trapèze, vide un sac de blé pour en sortir sa veste, casse une cruche pour y trouver son chapeau, etc. Dans cette séquence d'actions déjà, les trucages jouent un rôle important et établissent un univers diégétique clairement non réaliste et passablement " incohérent ».

La description du Bulletin Pathé évoque alors un rapport avec l'actualité (" les exploits des Carpentier et des Willie Lewis »), d'où sans doute aussi les images « sérieuses » d'un match

10 Voir Henri Bousquet, Catalogue Pathé des années 1896 à 1914. 1912-1913-1914, Éditions Henri Bousquet, 1995, p. 604. Le film sort en novembre 1912.

11 Le titre du film est Incohérences de Boireau, sorti en décembre 1912. Pour une description, voir ibid., p. 614. 
que l'on voit avant que Boireau ne se mette à prendre des leçons. Mais à proprement parler, Boireau ne pratique pas, dans ce film, le sport de manière un tant soit peu régulière. Après avoir donné un coup à l'instructeur, il descend dans la rue pour y frapper un malheureux passant, et ensuite aussi les deux messieurs qui l'ont contrarié auparavant. La boxe, autrement dit et comme dans le film avec Little Moritz, ne sert finalement que de prétexte pour motiver un certain nombre d'effets comiques basés, somme toute, sur un comportement agressif violant les règles sociales habituelles.

Dans ces deux films, il y a comme une tension comique entre l'activité sportive et les corps burlesques qui s'y adonnent. Little Moritz, par son comportement, fait rire les athlètes, et son style de boxe est très peu orthodoxe. Pour ce qui est du film avec André Deed, dès le début I'univers diégétique construit autour du personnage est caractérisé par l'absurdité et l'« incohérence ", qui se prolongent aussi au niveau de la boxe, notamment par le recours aux trucages pour montrer les effets des coups. Le corps de Boireau n'est littéralement pas à la hauteur de ses adversaires, mais cela est largement compensé par l'agressivité qui se déchaîne en lui après son " entraînement ". De la sorte, ce film s'inscrit dans la série des Boireau fondée sur un thème comique récurrent où le personnage est mis " hors de lui ", et se met dès lors à agresser tous ceux qu'il rencontre.

Fig. 3. Boireau, roi de la boxe, Pathé, 1912.

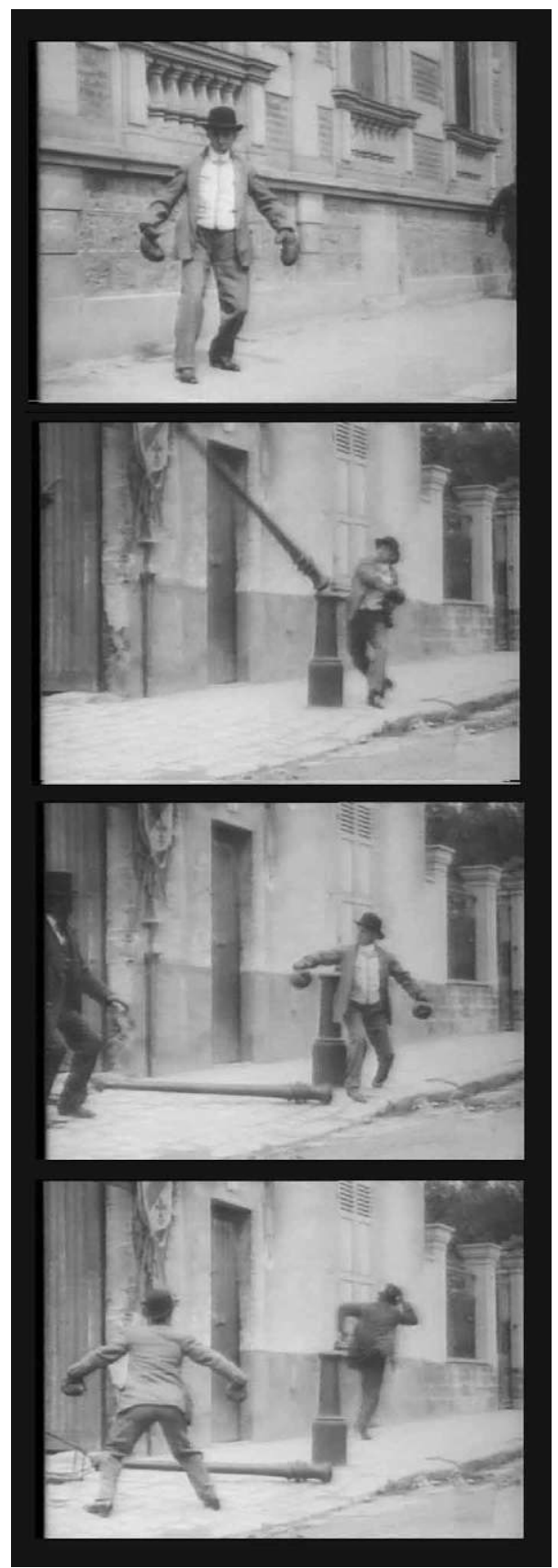




\section{Ruses et transgression des règles}

Tout comme Little Moritz, le personnage de Max est amené à pratiquer le pugilat afin de se voir accorder la main d'une jeune femme. Mais dans Boxeur par amour, la pratique du sport joue un rôle différent, comme le spécifie le résumé de Pathé :

Éperdu d'amour pour la fille du champion Jack Jeffroies, Max demande sa main. Mais trois prétendants sont déjà sur les rangs et Miss Jeffroies, qui est la meilleure élève de son père, n'accordera sa main qu'à son vainqueur dans un assaut de boxe. Le pauvre Max, entièrement novice dans cet art, se fait la main sur son domestique, qui n'ose riposter et notre ami, encouragé par ce premier résultat, n'hésite pas à se mesurer avec la jeune sportswoman qui a déjà mis knockout ses autres prétendants. Max subirait le même sort que ses rivaux, s'il n'avait l'idée ingénieuse d'employer une méthode aussi élégante qu'inédite en affolant sa partenaire par des " kisses » répétés. Pour la première fois, Miss Jeffroies est knock-out et ne se plaint pas de sa défaite. ${ }^{12}$

Dans ce film, Max pratique donc la boxe d'abord avec son domestique qui, lui, ne réagit pas à l'assaut, ce qui amène le héros à se méprendre sur ses propres capacités en la matière. Dans un deuxième temps, il doit affronter la « sportswoman » qui risque de le mettre knock-out, comme elle l'avait fait avec tous les autres prétendants. C'est par une ruse consistant à subvertir la pratique sportive par des actes amoureux que Max triomphe à la fin. La boxe en tant que sport joue par conséquent un rôle autrement important dans ce film, si l'on le compare aux exemples discutés ci-dessus. Au niveau de la description du film, on remarque que le nom du champion Jack Jeffroies (à part de jouer sur l'expression "j'effrois ») évoque celui de l'exchampion James Jeffries qui, le 4 juillet 1910 avait perdu le « match du siècle » contre le premier champion noir, Jack Johnson ${ }^{13}$.

Le fait que la protagoniste est une « sportswoman » qui défie les prétendants rend les choses plus complexes. La jeune Américaine est apparemment plus habile dans la pratique du sport que ses adversaires masculins, ce qui constitue déjà un premier niveau de transgression du cadre des attentes. Ce renversement des rôles des sexes, motivé ici par le fait qu'elle est la descendante d'un champion de boxe, produit un premier ressort du comique. Par ailleurs, si l'on en croit la description proposée par Pathé, les deux rencontres autour desquelles se construit le film apparaissent peu « sérieuses ». Le domestique refuse tout simplement de participer à

12 Ibid., p. 594. Le film sort en octobre 1912. Nous n'avons malheureusement pas pu consulter de copie de ce film et ne savons donc pas dans quelle mesure cette description est fiable.

13 En fait, le frère de James Jeffries, Jack Jeffries avait déjà perdu contre Johnson en 1902. Mais étant donné que sa carrière était terminée depuis longtemps en 1912, la référence à James Jeffries est la plus probable. Peut-être même que le nom dans la description a été conçu pour évoquer à la fois Johnson et Jeffries. 
l'effort sportif, et en face de la jeune femme, Max, craignant la défaite, ne respecte plus les règles du sport. Mais contrairement aux films avec Boireau et Little Moritz, Max n'agresse personne de manière sauvage. En transgressant les règles du sport, il renverse en quelque sorte les positions : tandis que la jeune femme demande à ses prétendants de s'affronter dans un combat de boxe au lieu de se comporter en amoureux, Max abandonne le rôle du sportif et réussit à susciter son désarroi justement par des gestes romantiques. Le changement de registre provoque à la fois le rire des spectateurs et, à un niveau diégétique, permet au personnage de Max de triompher ${ }^{14}$. Linder intègre encore la boxe dans un autre film, Max pratique tous les sports (fig. 4) qui sort en juillet 1913. De nouveau c'est une jeune Américaine qui promet sa main au vainqueur dans une compétition sportive :

Une charmante New-Yorkaise, Gladys Maxence, jolie comme savent l'être nos voisines d'Outre-Atlantique, passe dans les journaux une annonce : " Riche Américaine épouserait jeune homme connaissant tous les sports. » Des champions de boxe, de natation, de tennis,

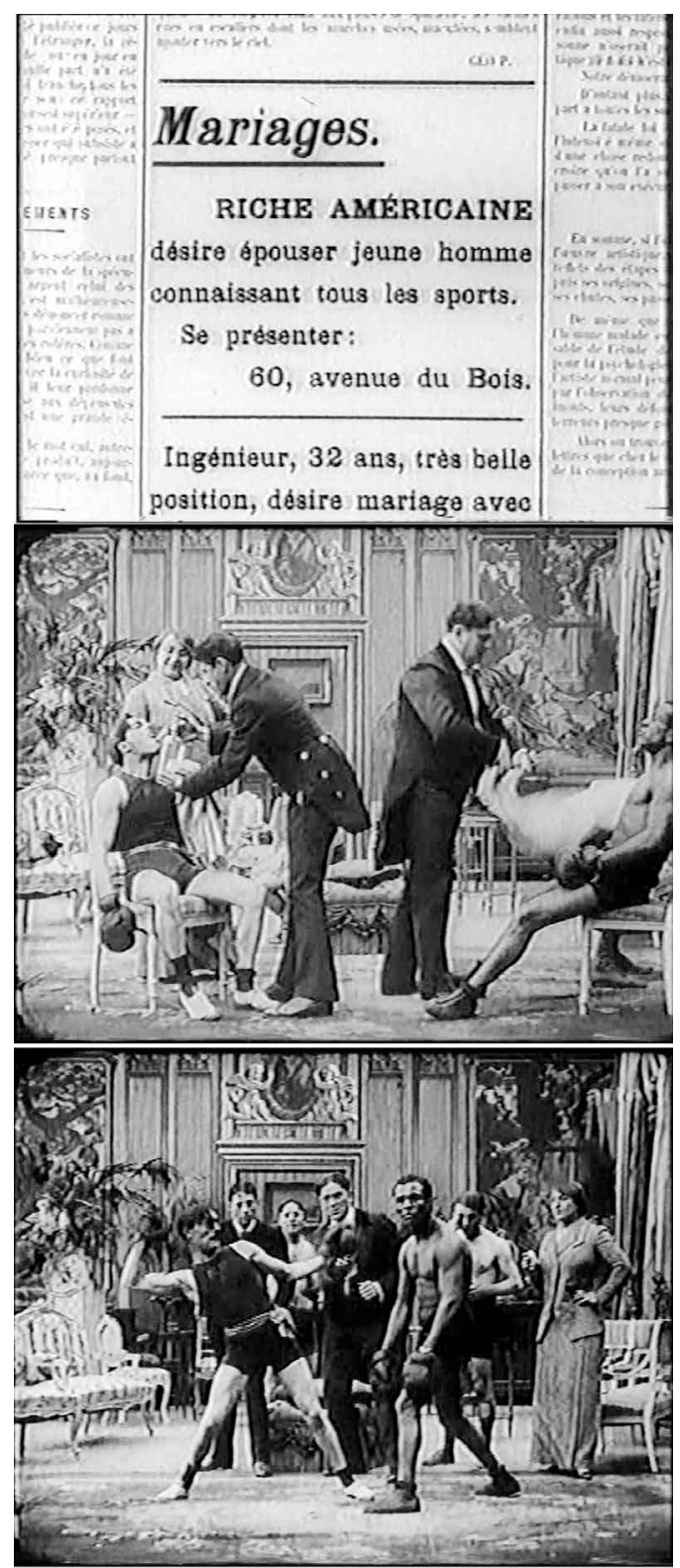

Fig. 4. Max pratique tous les sports, Pathé, 1913.

14 Le chassé-croisé entre le sport et l'amour, entre les différents rôles tenus par les sexes ainsi que les allusions que peut provoquer cette constellation narrative, mériteraient une analyse plus approfondie qui, cependant, dépasserait le cadre de la présente recherche. 
de golf, d'automobile, d'aéroplane : I'Irlandais Mac'Aron, le nègre Hiphiphongrah, I'Américain Sam Botte, le Roumain Couchetoilawa, l'Anglais Willy et le Français Max Linder, champion de tous les sports se mettent sur les rangs. La jeune Américaine ne sachant, entre tous les prétendants distingués, lequel choisir, organise un concours de tous les sports. Le vainqueur sera I'homme élu. Max triomphe à l'épreuve hippique et à l'escrime. Mais l'Anglais Willy le bat au skating. Max se rattrape au saut à la perche : vainqueur à $4 \mathrm{~m} 5$, mais au match de boxe, son excès d'ardeur le fait disqualifier. Heureusement, il bat brillamment tous ses concurrents dans la course d'avions, et Max et Willy se trouvant à égalité, sont seuls qualifiés pour disputer le dernier essai : l'épreuve automobile. La lutte est chaude. Notre héros, distancé par son rival, fait une imprudence et son auto fait panache. Il se relève sans mal, mais désespéré d'avoir perdu. Mais sa diplomatie supplée à sa malchance. Il apitoie la jeune Américaine en se disant grièvement blessé et gagne le charmant enjeu de ce moderne tournoi. ${ }^{15}$

Dans cette bande, considérablement plus longue que celles que nous venons d'évoquer, la pratique des sports devient un motif central, mais sans que le film cherche à créer un quelconque suspense. Le film met en valeur les qualités sportives de Max Linder (du personnage aussi bien que de l'acteur, pourrait-on dire), notamment en tant qu'escrimeur élégant et athlète passant la barre au saut à la perche ${ }^{16}$. Le potentiel comique des divers exercices, par contre, n'est pratiquement pas exploité. C'est seulement le skating qui provoque le rire sur les activités des sportifs, car ils ne cessent de tomber les uns sur les autres. Ils se montrent extrêmement maladroits sur leurs patins à roulettes, ce qui contraste d'autant plus avec les costumes et les chapeaux de soie qu'ils portent.

L'épreuve de la boxe représente une partie relativement longue du film. Elle a lieu dans le salon de Gladys Maxence. Dans une première rencontre, Hiphiphongrah triomphe puis Max réussit également à mettre son opposant par terre. Le match décisif oppose alors les deux vainqueurs. Au premier round, aucun des deux ne parvient à s'assurer un avantage sur l'autre. Le combat paraît assez réaliste, aucun effet comique n'intervient dans les actions des boxeurs. Après le premier round, à l'intervalle, ils s'assoient sur des chaises et les domestiques de Mlle Maxence s'occupent d'eux. À la toute fin de cette scène, Max émet un jet d'eau de sa bouche, mouillant l'homme qui est en train de lui enlever la sueur à l'aide d'une serviette. Cet interlude comique annonce en quelque sorte le changement de registre de la prochaine scène qui est précédée par l'intertitre : « 20e ROUND. Max est disqualifié ». De la sorte, il ne

15 Henri Bousquet, op. cit., p. 666.

16 En fait, on ne voit Linder que traverser la barre à une hauteur d'environ $2 \mathrm{~m}$. Pour le saut à $4 \mathrm{~m} 5$ dont parle la description du film et qui est annoncé dans un intertitre, on a recours au montage, avec un plan intercalé qui ne permet pas de percevoir la hauteur réelle. 
peut y avoir aucun suspense quant à l'issue du combat. Les deux adversaires sont visiblement au bout de leurs forces, leurs coups n'ont plus d'effet sur l'autre, et ils ont de la peine à rester debout. Un coup raté de Max frappe l'un des domestiques dans le visage, et l'homme s'écroule par terre. Un peu plus tard, Max essaie d'employer une ruse : il commence à rire et montre de sa main quelque chose derrière le dos de Hiphiphongrah. Quand celui-ci se retourne, Max rassemble toutes ses forces et frappe son adversaire, ce qui provoque alors sa disqualification.

La rencontre se termine alors de manière quelque peu burlesque. C'est encore une fois la transgression des règles qui provoque le rire, fût-ce, dans le cas présent, dans le cadre d'un combat de boxe qui, par ailleurs, est représenté de manière plutôt réaliste et sérieuse. Mais la transgression est ici motivée, pour ainsi dire, par la situation diégétique. C'est la longueur excessive de la rencontre et l'épuisement qui s'en suit qui amène Max à se servir de cette ruse (que la description du bulletin appelle « un excès d'ardeur »). Cela étant, ce comportement est en quelque sorte en accord avec le personnage de Max créé, non pas tant au sein de ce film particulier, mais au niveau de la série. C'est, après tout, une action « typiquement Max » qui, ici, le fait à la fois triompher et en même temps perdre le match. Qu'il réussisse, à la fin, " de gagner l'enjeu de ce moderne tournoi " grâce à une autre ruse, confirme justement cette image d'un Max avant tout très débrouillard, même s'il n'arrive pas toujours à parvenir au but. Ainsi le film se situe tout à fait dans la lignée de la série autour du personnage de Max. Mais en le comparant à d'autres, force est de constater qu'ici les effets comiques sont considérablement moins mis en avant que ne le sont les capacités sportives du héros aussi bien que de l'acteur. Ainsi, le corps de Max Linder est en effet présenté, dans Max pratique tous les sports du moins, comme un corps de sportif expert. II n'y a, contrairement aux films autour de Little Moritz et Boireau, rien de burlesque dans les mouvements de Max ${ }^{17}$. L'humour naît de transgressions qui jouent à un niveau différent.

\section{Diversité, diversification}

Au vu de films évoquant de manière diverse le thème de la boxe, on pourrait alors assez facilement concevoir une sorte d'« évolution » allant d'un comique basé sur des actions excessives, voire carrément absurdes, de la part des protagonistes, ainsi que sur le plaisir de la destruction et de l'agression, à des effets plus subtils jouant avant tout sur la transgression des règles du sport. Un tel développement irait alors clairement à l'encontre de l'observation de Jasset citée plus haut et qui voyait Max Linder comme un prédécesseur d'André Deed. Or,

17 À l'exception, bien évidemment, du concours de patins à roulettes. 
souscrire à une telle perspective reviendrait à oublier que tous les films pris en considération ici sortent entre octobre 1912 et juillet 1913, donc quasiment à la même époque et, de surcroît, dans un ordre qui ne permet guère de déterminer une « évolution » quelconque. Si I'on ajoute encore, à ces exemples tirés de la production Pathé, les films de la série autour du personnage de Léonce incarné par Léonce Perret, l'éventail des stratégies du comique s'élargit encore plus ${ }^{18}$. Évidemment, Léonce (tout comme, probablement, Rigadin au sein de la maison Pathé) ne s'engagerait jamais dans des exploits sportifs comme la boxe. Mais ceci, justement, témoigne de la diversité des types de comique existant dans le cinéma français au début des années 1910. En multipliant les options pour les exploitants, les maisons de production, manifestement, misent sur la diversification à travers la diversité. Et, par conséquent, il serait en effet intéressant de creuser la question en tenant compte aussi de la différentiation au niveau des lieux de projection ${ }^{19}$. Est-ce que ces comiques se trouvent représentés de manière égale dans les programmes des différents types d'établissements ? Y a-t-il une répartition au niveau des préférences selon le critère des couches sociales ? Selon les quartiers, par exemple dans une ville comme Paris ? À quoi, exactement, correspond une telle stratégie de production au niveau de la distribution et de l'exploitation des films ? Une historiographie tenant compte des seuls critères esthétiques ne se préoccupera guère de telles questions. Mais pour comprendre le fonctionnement historique du cinéma comique au début des années 1910, elles sont essentielles.

Pour en revenir à Victorin Jasset : c'est probablement le fait qu'il cherche à décrire l'évolution du spectacle cinématographique comme une succession d'écoles qui détermine son point de vue de telle manière qu'il renverse en quelque sorte la chronologie. En déclarant que Deed « avait fait école, presque en même temps que Max Linder, son précurseur ", il constate en fait avant tout une quasi-contemporanéité des deux comiques. Mais, à cause de la perspective qu'il adopte, il lui faut établir une certaine filiation. Et du point de vue d'un observateur de 1911, on pouvait peut-être avoir eu l'impression que Deed " avait fait école » un peu plus que Linder. Cela, en fin de compte, démontre aussi comment sont relatifs les jugements de cet ordre, car chaque époque se construit ses propres visées. D'où la nécessité d'interroger et de réinterroger I'historiographie.

18 Voir Laurent Le Forestier, "Les comédies de Léonce Perret, fleurons de la production Gaumont ", dans Léonce Perret (sous la direction de Bernard Bastide et de Jean A. Gili), Paris, AFHRC / Cineteca di Bologna, 2003, pp. 28-48.

19 Je remercie Sabine Lenk de m'avoir suggéré cette piste d'interrogation. 